\title{
The Influence on Numerical Taxonomic Similarities of Errors in Microbiological Tests
}

\author{
By P. H. A. SNEATH AND R. JOHNSON \\ MRC Microbial Systematics Unit, University of Leicester, and National Collection of \\ Type Cultures, Central Public Health Laboratory, Colindale, London, N.W.9
}

(Accepted for publication 28 April 1972)

SUMMARY

A study is made of the effect of experimental errors of microbiological tests upon similarity values used in numerical taxonomy. For the coefficient $S_{\mathrm{SM}}$ approximations are given for the mean and standard error of similarity values when some test results are erroneous, $S^{\prime}$, if the true value of similarity $(S)$, the average probability in I, o data of erroneous tests results $(p)$, and the number of tests $(n)$ are known. Formulae are given for estimating $p$ from an analysis of variance, and for including or excluding chosen factors that affect test reproducibility.

When $p$ is over about $10 \%$ the error of similarity values becomes unacceptably large. Test error also increases the existing sampling error. It is generally better to employ many tests even if they are not as reproducible as desired, rather than to use only a few extremely reproducible tests.

Examples are given suggesting that within one laboratory $p$ can usually be kept below $5 \%$, but the discrepancies between laboratories may often be much larger. It is advisable to replicate some of the strains in numerical taxonomic studies, so as to afford a check on test reproducibility.

\section{INTRODUCTION}

It is becoming increasingly realized that experimental errors in conventional microbiological tests are usually quite appreciable even under conditions of careful standardization (e.g. Gottlieb, 196r ; Lapage, Bascomb, Willcox \& Curtis, 1970; Taylor, Guthrie \& Shirling, 1970; Report of the Pseudomonas Working Party, to be published). It is also becoming clear that such errors introduce corresponding uncertainties into numerical taxonomies. Lockhart (1967) and Sneath (I97I) have drawn attention to the need to improve standardization of tests for this reason, and the problem is now becoming of interest in plant chemotaxonomy and in virus taxonomy (e.g. Taylor \& Campbell, 1969; Tremaine \& Argyle, 1970).

Little is yet known, however, about the magnitudes of such errors in ordinary work, and it is the purpose of this paper to report on this and discuss suitable methods of estimating them. A full examination of factors responsible for lack of reproducibility is given in the Report of the Pseudomonas Working Party (to be published), and therefore in the present paper the effect of error on similarity values will be investigated, directed mainly to situations likely to occur in numerical taxonomic work. This will principally concern the reproducibility within one laboratory under the same conditions (for which data are now becoming available), but brief attention will be given to discrepancies between results under different conditions or in different laboratories. Test error has the general effect of lowering similarities between phenetically similar strains, increasing the scatter and uncertainty of similarity values, and making clusters less compact (see Fig. I). 


\section{METHODS}

Effect of test errors on $S_{\mathrm{SM}}$

The statistical effect of test errors on the similarity, $S$, between two strains, $j$ and $k$, has been explored for presence-absence tests and the coefficient $S_{\mathrm{SM}}$ (see Sokal \& Sneath, 1963) as follows. The conventional symbolism of a $2 \times 2$ table is adopted, where $a, b, c$ and $d$ are respectively the number of tests in which both strains $j$ and $k$ are positive (scored $\mathrm{I}, \mathrm{I}$ ), negative in $j$ and positive in $k(0, \mathrm{I})$ positive in $j$ and negative in $k(\mathrm{I}, 0)$ and negative in both $(0,0)$. The total of $a+b+c+d$ is $n$, and $S_{\mathrm{SM}}$ is $(a+d) / n$. Let $p$ be the probability that a result is erroneous, averaged over the $n$ tests, taking it as equally probable that a negative will be misread as a positive and vice versa, and also assuming that the probabilities are the same for both strains. These are simplifying assumptions which are likely to hold reasonably well in practical applications, but some exceptions are briefly considered in the discussion.

The effect of the errors is to shift the value of $S$ that would have been obtained from errorfree tests to a new value $S^{\prime}$, that lies in general nearer to 0.5 ; it also introduces scatter, so that the value of $S^{\prime}$ that will be observed is uncertain within certain limits. This is because, due to chance, there will not always be exactly the number of errors expected. Using some simplifying statistical assumptions the following approximation was derived (Sneath, I97I) for the expected (mean) value of $S^{\prime}$.

$$
\mathrm{E}\left(S^{\prime}\right) \simeq S(2 p-\mathrm{I})^{2}+2 p(\mathrm{I}-p) .
$$

The variance of $S^{\prime}$ is difficult to estimate by simple approximations. One such rough estimate has been given (Sneath, I97I) but, several other ways of deriving it from binomial and Poisson distributions give appreciably different results in some parts of the range of $S$ or $p$. The mean and variance were therefore checked by computer simulation using random numbers, which showed that formula (I) gave a good approximation for the mean. It also showed that the variance was close to $2 p(\mathrm{I}-p) / n$ as noted by Sneath (I97I), but that the approximation given there does not fit too well near $S=0.5$ or $p$ over 0.1 , because it does not take into account the increased variance due to a difference of proportions, or the deviation of the Poisson distribution from the binomial at high values of $p$. These have been allowed for in the formula given below for the standard error of $S^{\prime}$ :

$$
\mathrm{SE}\left(S^{\prime}\right) \simeq \sqrt{2 p(\mathrm{I}-p) / n} \times \sqrt{\mathrm{I}-2 p(\mathrm{I}-p)} .
$$

The first term is derived as follows for small $p$ and large $n$ : the total number of changes introduced by test error will approximate a Poisson distribution. This has both mean and variance equal to $2 p(\mathrm{I}-p) n$; of these a mean proportion $S$ will be changes moving $S$ toward 0.5 and a mean proportion ( $\mathrm{I}-S$ ) will be changes moving $S$ toward I or 0 . If these are taken as independent Poisson distributions, then the variance of their difference will equal their sum, i.e. $2 p(\mathrm{I}-p) n$. The second term is introduced because when $p$ is large (over about $0^{\circ} \mathrm{I}$ ) the distribution deviates substantially from a Poisson distribution, and this term is obtained from the analogous binomial distribution $2 p(\mathrm{I}-p)[\mathrm{I}-2 p(\mathrm{I}-p)] n$. When expressed as a proportion this yields the standard error given by formula (2) above. The computer simulations showed close agreement with this formula over most of the ranges of $S, p$ and $n$, closer than for the other approximations tried. As expected, skewness and kurtosis were very small (except for very small values of $p$ and $n$ ).

For reasons given later, it will be convenient to calculate these quantities from the pooled variance of the tests, $s^{2}$ (not to be confused with the variance of $S^{\prime}$, which is the square of 
Table I. Values of $E\left(S^{\prime}\right)$ for various values of $p$ and $S$

For $S$ below 0.5 , calculate $\mathrm{I}-S$, and enter the Table with this when one obtains $\mathrm{I}-\mathrm{E}\left(S^{\prime}\right)$.

\begin{tabular}{|c|c|c|c|c|c|c|c|c|c|}
\hline$S$ & 0.001 & 0.01 & 0.02 & 0.05 & $0 \cdot \mathbf{I}$ & 0.2 & 0.3 & 0.4 & 0.5 \\
\hline 1.0 & 0.9980 & 0.9802 & 0.9608 & 0.9050 & 0.8200 & 0.6800 & 0.5800 & 0.5200 & 0.5000 \\
\hline 0.9 & 0.8984 & 0.8842 & 0.8686 & 0.8240 & 0.7560 & 0.6440 & 0.5640 & 0.5160 & 0.5000 \\
\hline 0.8 & 0.7988 & $0.788 \mathrm{I}$ & 0.7765 & 0.7430 & 0.6920 & 0.6080 & 0.5480 & 0.5120 & 0.5000 \\
\hline 0.7 & 0.6992 & $0.692 \mathrm{I}$ & 0.6843 & 0.6620 & 0.6280 & 0.5720 & 0.5320 & 0.5080 & 0.5000 \\
\hline 0.6 & 0.5996 & 0.5960 & 0.5922 & $0.58 \mathrm{I} 0$ & 0.5640 & 0.5360 & 0.5160 & 0.5040 & 0.5000 \\
\hline 0.5 & 0.5000 & 0.5000 & 0.5000 & 0.5000 & 0.5000 & 0.5000 & 0.5000 & 0.5000 & 0.5000 \\
\hline
\end{tabular}

Table 2. Standard error of $S^{\prime}$

\begin{tabular}{|c|c|c|c|c|c|c|c|c|c|}
\hline$n$ & $0.00 \mathrm{I}$ & 0.01 & 0.02 & 0.05 & $0 \cdot I$ & 0.2 & $0 \cdot 3$ & 0.4 & 0.5 \\
\hline IO & $0.014 \mathrm{I}$ & $0.044 \mathrm{I}$ & 0.0614 & 0.0927 & 0.1215 & 0.1475 & 0.1561 & O. I 580 & $0.158 \mathrm{I}$ \\
\hline 20 & 0.0100 & 0.0312 & 0.0434 & 0.0656 & 0.0859 & $0 \cdot 1043$ & 0.1104 & O. I I I 7 & 0.1118 \\
\hline 50 & 0.0063 & 0.0197 & 0.0274 & 0.0415 & 0.0543 & 0.0660 & 0.0698 & 0.0707 & 0.0707 \\
\hline 100 & 0.0045 & 0.0139 & 0.0194 & 0.0293 & 0.0384 & 0.0466 & 0.0494 & 0.0500 & 0.0500 \\
\hline 200 & 0.0032 & 0.0099 & 0.0137 & 0.0207 & 0.0272 & 0.0330 & 0.0349 & 0.0353 & 0.0354 \\
\hline 500 & 0.0020 & 0.0062 & 0.0087 & 0.0131 & 0.0172 & 0.0209 & $0.022 \mathrm{I}$ & 0.0223 & 0.0224 \\
\hline 1000 & 0.0014 & 0.0044 & $0.006 \mathrm{I}$ & 0.0093 & $0.012 \mathrm{I}$ & 0.0148 & 0.0156 & 0.0158 & 0.0158 \\
\hline
\end{tabular}

the standard error mentioned above). This can be done because of the following relations between the variance $s_{i}^{2}$ and the proportion of errors, $p_{i}$, for a presence-absence test $i$.

$$
\begin{gathered}
s_{i}^{2}=p_{i}\left(\mathrm{I}-p_{i}\right), \\
p_{i}=\frac{1}{2}\left(\mathrm{I}-\sqrt{\mathrm{I}-4 s_{i}^{2}}\right) .
\end{gathered}
$$

Then, in place of formula (I) and formula (2) respectively we may use

$$
\begin{aligned}
\mathrm{E}\left(S^{\prime}\right) & \simeq S\left(\mathrm{I}-4 s^{2}\right)+2 s^{2}, \\
\mathrm{SE}\left(S^{\prime}\right) & \simeq \sqrt{2 S^{2}\left(\mathrm{I}-2 s^{2}\right) / n} .
\end{aligned}
$$

It should be noted that $p_{i}$ is to some extent a convenient mathematical fiction, in that when $s_{i}^{2}$ is over 0.25 this corresponds to imaginary values of $p_{i}$. This is a consequence of the fact that $s_{i}^{2}$ can in theory reach 0.5 if a single pair of replicates is made, because the degree of freedom is then only I, but in practice $s_{i}^{2}$ will seldom reach this maximum. The reason for this is that when discrepancies are maximal there will generally be, on the average, as many pairs which by chance are scored the same (I, I or $\mathrm{O}, 0)$ as show discrepancies $(\mathrm{O}, \mathrm{I}$ or $\mathrm{I}, \mathrm{O})$, so that the maximum value of $s_{i}^{2}$ will, with a large set of strains, average 0.25 . This is only likely to be exceeded if a test is predominantly positive on one occasion and then, because of some gross technical failure, is predominantly negative on the other. In such cases the formulae (I) to (6) will be misleading, and the data cannot be used for any worthwhile study until the technique has been re-examined.

In the majority of practical applications $S$ will be lowered; $S$ values below 0.5 will be raised, but these are of less interest in numerical studies. Table r shows a selection of $\mathrm{E}\left(S^{\prime}\right)$ values from formula (I), from which it can be seen that for $p$ less than about $0 \cdot I$ the disturbance of $S$ is not very great, resulting in a lowering of $S$ (on the average) by $2 p$, or some- 

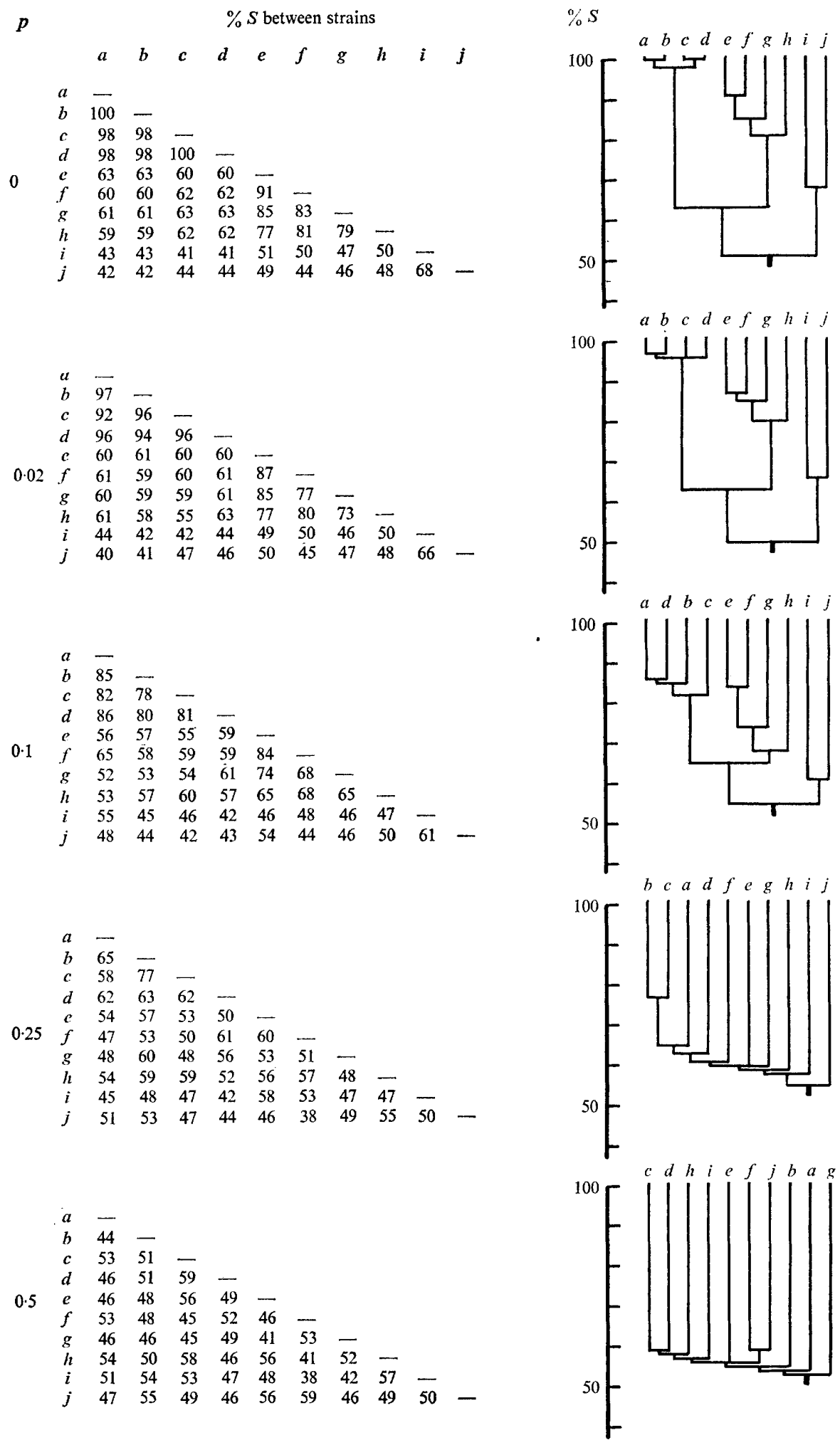
what less, for the range of $S$ of greatest interest, i.e. $0 \cdot 7$ to $\mathrm{I} \cdot 0$. Thus, for example, if $p$ is 0.05 , or $5 \%$, a pair of identical strains (such as 2 subcultures of I isolate) will usually have an observed similarity, $S^{\prime}$, of about 0.9 , or $90 \%$. In Table 2 is given similar information, based on formula (2), for the standard error of $S^{\prime}$, from which the scatter of $S^{\prime}$ can be estimated for typical values of $p$ and $n$; for other values interpolation can be used. The standard errors can be used to estimate the likely bounds of $S^{\prime}$, because $S^{\prime}$ would be expected to lie between

$$
\mathrm{E}\left(S^{\prime}\right) \pm x \mathrm{SE}\left(S^{\prime}\right)
$$

where $x$ is the number of standard errors of the normal distribution corresponding to the two-tailed confidence limits desired. Thus for the example above, if $n$ was 100, the value of $S^{\prime}$ would be expected to lie on $95 \%$ of occasions between $0.9050 \pm \mathrm{I} \cdot 96(0.0293)$, i.e. between $84.76 \%$ and $96.24 \%$. These approximations are not suitable if $\mathrm{E}\left(S^{\prime}\right)$ is very close to o or $\mathrm{I}$, when the arcsine transformation should be considered (see Goodall, 1967; Owen, I962, p. 293). These formulae also allow some estimate of the maximum test error that can be tolerated if it is required to know $S^{\prime}$ to some given degree of accuracy. It may be concluded that for most work a value of $p$ up to $5 \%$ will have little detrimental effect, but if it rises above about Io $\%$ the consequences may be serious. An illustration on a hypothetical example is shown in Fig. $I$.

\section{Relation of test error to sampling error}

In most applications the test error increases the uncertainty of estimate of a given similarity value above the existing uncertainty due to sampling error (Sneath, 197I). The sampling variance, due to the choice of a particular selection of $n$ tests, is approximately

$$
\mathrm{SE}_{\text {sampling }}^{2} \simeq S(\mathrm{I}-S) / n \text {. }
$$

This is the familiar binomial variance, and Goodall (1967) has shown that it is a reasonable approximation to the sampling error of $S_{\mathrm{SM}}$. It seems unlikely that the test error would be appreciably correlated with the sampling error, so one could sum the two variances to obtain more realistic confidence limits to $S$ values against which to judge the significance of observed differences between strains or clusters. This can be expressed as

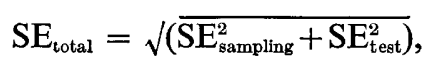

where $\mathrm{SE}_{\text {samping }}^{2}$ is given by formula (8) and $\mathrm{SE}_{\text {test }}^{2}$ by squaring the standard error from formula (2) or formula (6). High values of $p$ will lead to the total error rising to unacceptable levels even if the number of tests is large, while if the number of tests is small these levels will be reached even more readily.

Fig. I. Effect of test error on similarity values and phenograms. A hypothetical example is given, based on Io strains, $a$ to $j$, score for 100 presence-absence characters. At the top of the Figure is shown the similarity matrix, and the single-linkage phenogram derived from it, when no test errors are present $(p=0)$. Successive stages below this show the effect of increasing the error to the maximum of $p=0.5$, by introducing random errors by a Monte Carlo technique.

It can be seen that the very compact cluster $a-d$, containing two identical pairs, is affected noticeably even by $2 \%$ of error, while at high-error levels the taxonomic structure of all clusters is severely disturbed.

As $p$ increases from 0 to 0.5 the correlation between the error-free matrix and those containing error falls $(r=\mathrm{I} \cdot 0,0.994,0.963,0.708$ and -0.039 respectively). The correlation between the phenograms and the similarity matrices from which they were derived (the cophenetic correlation) also falls (respectively $r=0.99 \mathrm{I}, 0.988,0.960,0.740$ and $0.4 \mathrm{II}$ ); this shows that the phenograms are increasingly poor summaries of the similarities, even taking into account the fact that these similarities are themselves more and more distorted. 

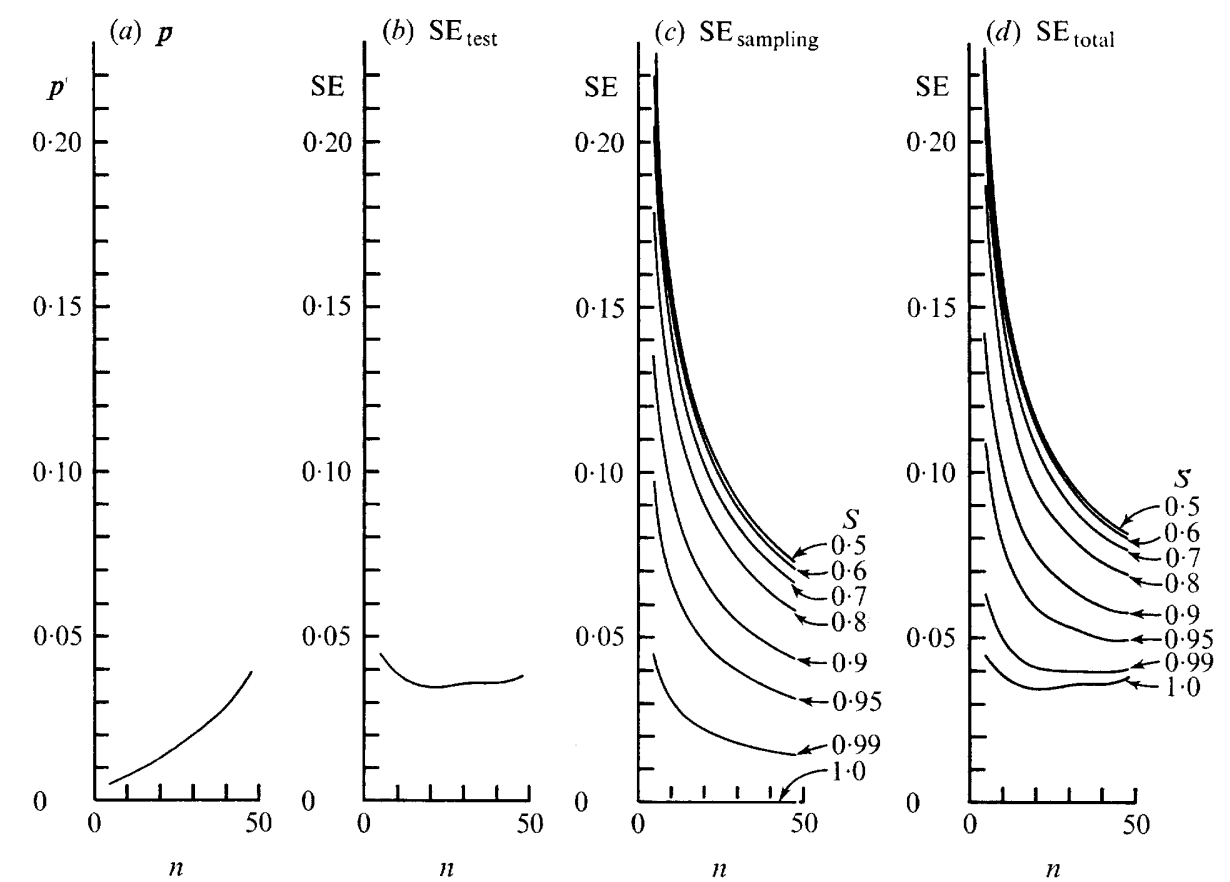

Fig. 2. Effect of including an increasing number of tests with poor reproducibility in the set used to estimate a similarity coefficient, beginning with the most reproducible tests. (illustrative example from the 48 tests of Table 9 in Lapage et al. 1970). (a) Value of $p$ at increasing levels of $n$, calculated from the pooled test variances; $(b)$, values of test error as $\mathrm{SE}_{\text {test }}$ for increasing $n$ (from values of $p$ in (a) and formula 2$) ;(c)$, values of sampling error as $\mathrm{SE}_{\text {sampling }}$ for increasing $n$ (from formula 8 ) and for different values of $S ;(d)$ values of total error as $\mathrm{SE}_{\text {total }}$ for increasing $n$ (from formula 9) and for different values of $S$.

One important consequence of formula (9) should be mentioned. The effect of including tests with high experimental error is not all loss; it is counterbalanced by the reduction of sampling error consequent on the increase in the number of tests. This is illustrated in Fig. 2, which shows the effect of including tests in turn in the set used to calculate similarity, starting with the most reproducible ones, and adding the others in increasing order of $p_{i}$ using the data of Lapage et al. (1970) as an illustration. The increase in test error due to including the more unreliable tests is at first more than compensated for by reduced sampling error. Only at very high values of $S$ near $\mathrm{I} \cdot 0$ does the total error, measured by $\mathrm{SE}_{\text {total }}$, begin to rise again while the tests are still few. At lower $S$ values the curve of the total error does not turn upward even on including the last and worst tests (with $p_{i}$ over $15 \%$ ). This behaviour is due to the small size of the binomial sampling error at high $S$ values (the sampling error is nominally zero when $S=\mathrm{I}$ ). In practice the sampling error will be somewhat greater than formula (8) suggests (see Owen, 1962, p. 293, whose tables suggest that a better estimate of $\mathrm{SE}_{\text {sampling }}$ when $S=\mathrm{I}$ is given by $\mathrm{I} / 4 n$ ), so this continued decline in total error with increasing $n$ is likely to be found even at high $S$.

It may be concluded that in practice it is better as a rule to include as many tests as possible unless they are extremely unreliable, rather than to employ only a few very reproducible tests. This point has been questioned in classifications in other fields (Williams \& Lance, I969) so it is worth noting. Another consequence of large $n$ is that the variance of $S^{\prime}$ becomes smaller as $n$ increases, so that the calculation of a corrected value discussed below becomes 
more reliable. Evidently numerical taxonomic studies can in practice tolerate a moderate amount of unreliable data. The work of Fisher \& Rohlf (I969) on miscoding of characters carries a similar implication. Nevertheless, tests with $p_{i}$ over about $0 \cdot 1$ must be a cause for concern, and warrant efforts at improvement in technique.

One cannot correct an observed similarity value for sampling error without a larger sample of tests (and even then there are theoretical and practical problems). But if we know $p$ can one correct for the test error? That is, can one correct an observed similarity value (i.e. $S^{\prime}$ ) to a better value, closer to that which would have been obtained from tests free from experimental error? This can only be done partially, because of the uncertainty introduced by the test error, and only usefully if $n$ is very large. If $n$ were large and if, for example, $p$ was known to be 0.05 , one could estimate the true value on that set of tests for the similarity between a pair of strains which had given an observed similarity of 0.83 . This would be about 0.907 , using the relation

$$
S=\left[S^{\prime}-2 p(\mathrm{I}-p)\right] /(2 p-\mathrm{I})^{2} .
$$

But it would only be somewhere near this figure. Thus if $n$ was only Io, $\mathrm{SE}_{\text {test }}$ would be so large, about $9 \%$, that one could not be at all sure if $S$ was close to 0.907 ; it could easily be $\mathrm{I} \cdot 0$ or 0.85 . Thus the correction, whilst it would give tighter clusters as a rule, would need to be used with a good deal of caution. Nevertheless, it could be argued that the uncorrected value would usually be almost as uncertain, and this correction principle has been successfully used in another context in estimating similarities in population genetics (e.g. Grewal, I962).

\section{Estimation of average probability of error}

The use of an average probability for test results, $p$, is based on the assumption that it will give values of $S^{\prime}$ acceptably close to those that would be obtained in a long series of similarity values calculated from tests which have in fact different error rates, $p_{1}, p_{2}, \ldots p_{i}, \ldots p_{n}$. The validity of this assumption rests on general grounds similar to those used to estimate the sampling error of $S_{\mathrm{SM}}$ and shown by Goodall (1967) to be acceptable for most practical purposes. The best way of calculating $p$ is not, however, to average the $n$ values of $p_{i}$ for individual tests, although for small values of $p_{i}$ the results will not be very inaccurate. It can be shown that a better value is given by averaging the individual test variances, $s_{i}^{2}$, to give the pooled variance, $s^{2}$ :

$$
s^{2}=(\mathrm{I} / n)\left(s_{1}^{2}+s_{2}^{2} \ldots+s_{i}^{2} \ldots+s_{n}^{2}\right)
$$

This can be seen from a hypothetical case of seven tests each with $p_{i}$ of 0.2 and 3 tests each with $p_{i}$ of 0.04 . The mean $p$ calculated by averaging the $p_{i}$ values is 0.152 . Taking $S$ to be 0.80 as an example, $\mathrm{E}\left(S^{\prime}\right)$ calculated from formula (I) is 0.6453 . In fact, the expected value of $S^{\prime}$ is made up of seven tests with $p_{i}=0.2$ (giving an expectation of an average of 4.2560 matches on these seven) plus three further tests with $\mathrm{p}_{i}=0.04$ (giving an expectation of an average of $2 \cdot 2618$ matches on these). The expected matches for the ro tests together is thus the sum $6.5 \mathrm{I} 78$, so that over the Io tests, $\mathrm{E}\left(S^{\prime}\right)$ will actually be $0.65 \mathrm{I} 78$. This value is identical with that obtained by using the pooled variance $s^{2}, 0 \cdot 12352$ (corresponding to $p$ of $0 \cdot 144360$ ). Similar considerations apply to the standard error of $S^{\prime}$. Formulae (5) and (6) can be used with $s^{2}$, or $s^{2}$ can be converted into $p$ using the relation given in formula (4).

The use of the test variances is also convenient in the practical assessment of test reproducibility. The usual form of data will be, for a given test $i$, a table of $t$ strains in rows against $N$ replicates in columns, done in the same laboratory (or more rarely, in different laboratories). The proportion of discrepancies can be used to calculate $p_{i}$, but for this one 
Table 3. Illustrative example of a set of test data and analysis of variance

(a) Test results

\begin{tabular}{|c|c|c|c|c|c|c|c|c|c|c|}
\hline \multirow[b]{2}{*}{ Strains } & \multicolumn{5}{|c|}{ I day readings } & \multicolumn{5}{|c|}{2 day readings } \\
\hline & \multicolumn{4}{|c|}{ Replicates } & $\hat{s}_{i j}^{2}$ & \multicolumn{4}{|c|}{ Replicates } & $\hat{s}_{i j}^{2}$ \\
\hline $\mathbf{I}$ & $\mathbf{I}$ & 0 & I & 0 & $1 / 3$ & I & 0 & I & 0 & $1 / 3$ \\
\hline 2 & I & 0 & 0 & I & $\mathrm{I} / 3$ & I & I & I & I & 0 \\
\hline 3 & I & I & I & 0 & $\mathrm{I} / 4$ & I & I & I & 0 & $\mathrm{I} / 4$ \\
\hline 4 & 0 & 0 & 0 & 1 & $1 / 4$ & 0 & 0 & 0 & I & $1 / 4$ \\
\hline 5 & 0 & 0 & 0 & 0 & 0 & 0 & 0 & 0 & 0 & 0 \\
\hline \multicolumn{5}{|c|}{ Averages $s_{i j}^{2}=s_{i}^{2}$} & $7 / 30$ & & & & & $5 / 3$ \\
\hline
\end{tabular}

(b) Analyses of variance for each time of reading separately

\begin{tabular}{|c|c|c|c|c|c|c|}
\hline \multirow[b]{2}{*}{ Factors } & \multicolumn{3}{|c|}{ I day readings } & \multicolumn{3}{|c|}{2 day readings } \\
\hline & $\begin{array}{c}\text { Sums of } \\
\text { squares }\end{array}$ & $\begin{array}{l}\text { Degrees of } \\
\text { freedom }\end{array}$ & Variance & $\begin{array}{r}\text { Sums of } \\
\text { squares }\end{array}$ & $\begin{array}{l}\text { Degrees of } \\
\text { freedom }\end{array}$ & Variance \\
\hline Between replicates & 0.40 & 3 & 0.1333 & 0.20 & 3 & 0.0667 \\
\hline Between strains & $I \cdot 30$ & 4 & 0.3250 & $2 \cdot 50$ & 4 & 0.6250 \\
\hline Residual & $3 \cdot 10$ & I 2 & 0.2583 & $2 \cdot 30$ & 12 & 0.1917 \\
\hline Total & $4 \cdot 80$ & 19 & 一 & $5 \cdot 00$ & 19 & - \\
\hline
\end{tabular}

(c) Analysis of variance for all factors together

\begin{tabular}{lccc}
\multicolumn{1}{c}{ Factors } & $\begin{array}{c}\text { Sums of } \\
\text { squares }\end{array}$ & $\begin{array}{c}\text { Degrees of } \\
\text { freedom }\end{array}$ & Variance \\
$\begin{array}{l}\text { Main factors } \\
\text { Between replicates }\end{array}$ & 0.50 & 3 & 0.1667 \\
Between strains & 3.40 & 4 & 0.8500 \\
$\quad$ Between times of reading & 0.10 & I & 0.1000 \\
Interactions & & & \\
$\quad$ Replicates $\times$ strains & 5.00 & 12 & 0.4167 \\
Replicates $\times$ times of reading & 0.10 & 3 & 0.0333 \\
Strains $\times$ times of reading & 0.40 & 4 & 0.1000 \\
Residual & 0.40 & 12 & 0.0300 \\
Total & 9.90 & 39 & -
\end{tabular}

needs to know the 'true' result, and, although this may be assumed to be the majority result, this conclusion is not certain if the number of replicates is finite. This problem is obvious when there are only two results on each strain: one normally cannot tell which is 'correct'. Only rarely will it be possible to compare a single replicate with a very accurate result based on an extremely well-standardized technique that can be taken as a true standard; if these conditions do hold, the variance calculated by formula (I2) below will underestimate the true variance, but the methods suggested below, which do not assume knowledge of a true standard, are better statistical practice in normal work. The problem can be circumvented by calculating an estimate of the variance of the row. Thus for strain $j$ one calculates

$$
\hat{s}_{i j}^{2}=\frac{\mathrm{I}}{N-\mathrm{I}} \Sigma\left(X-\frac{\Sigma X}{N}\right)^{2},
$$

where $\hat{s}_{i j}^{2}$ is the best estimate of the unknown population variance $\sigma_{i j}^{2}, X$ is here o or I, and $N-\mathrm{I}$ is the degrees of freedom. For each strain one computes $\hat{S}_{i j}^{2}$ and takes the average over the strains to obtain $s_{i}^{2}$ for use with formula (II).

While this method gives an answer for $s_{i}^{2}$, it requires $t$ separate calculations of variance. 
It is much quicker to obtain $s_{i}^{2}$ from an analysis of variance. The technique is described in standard textbooks of statistics (e.g. Moroney, 1956; Snedecor, 1956; Sokal \& Rohlf, 1969) where the actual computation methods can be found.

An illustration is shown in Table 3. Considering first the results at $\mathrm{I}$ day, the variation between strains should not be counted for the present purpose as unwanted variation. If therefore from the total sum of squares one subtracts the sum of squares attributable to differences between strains, one obtains that due to other causes (in this case, differences in replicates plus erratic variation not attributable to difference in the average behaviour of strains or of replicate tests). This remaining sum of squares, divided by the total degrees of freedom minus the degrees of freedom of the strains, gives $s_{i}^{2}$, i.e.

$$
s_{i}^{2}=\frac{\text { Sum of squares (total-strains) }}{\text { Degrees of freedom (total }- \text { strains) }} .
$$

In this example for the I day readings it is, from Table $3(b),(4 \cdot 80-\mathrm{I} \cdot 30) /(19-4)=3 \cdot 50 / \mathrm{I} 5$, which is identical to the average $s^{2}$ found by averaging the variances for the strains and shown in Table $3(a)$ in the form $7 / 30$. For a case such as this the calculation of $s_{i}^{2}$ is even simpler, and there is no need to carry out a complete analysis of variance, because for $\mathrm{I}$, $o$ data one need only calculate

$$
s_{i}^{2}=\left[\text { Grand total }-\frac{\mathrm{I}}{N} \Sigma\left(\text { strain totals }{ }^{2}\right)\right] / t(N-\mathrm{I})
$$

When a single pair of replicates is performed, this formula simplifies even further, to

$$
s_{i}^{2}=d / 2 t,
$$

where $d$ is the number of strains giving discrepant results in the two series.

This method can also be readily adapted to more involved analyses of variance, as illustrated by Table $3(c)$, where the variables are strains, replicates and times of reading. If one wishes to obtain an estimate of the variation over strains in different replicates but averaged over the two times of reading one can calculate

$$
s_{i}^{2}=\frac{\text { Sums of squares (total }- \text { strains }- \text { times }- \text { strains } \times \text { times interaction) }}{\text { Degrees of freedom (total }- \text { strains }- \text { times }- \text { strains } \times \text { times interaction) }} .
$$

In the example this is $(9 \cdot 9-3 \cdot 4-0 \cdot I-0 \cdot 4) /(39-4-I-4)$, which is $6 \cdot 0 / 30$, and this can be seen to be the mean of the separate average $s^{2}$ values, $7 / 30$ and $5 / 30$, for the two times of reading in Table $3(a)$.

Different combinations of sums of squares and degrees of freedom may be chosen to estimate the mean variance that would be expected from matched pairs of test results under some other desired condition. Thus, if one wished to know the mean variance one would obtain by comparing each I day reading with its corresponding 2 day reading (as might be needed to investigate the effect of reading tests on some strains at a different time from those on other strains) this could be found as the sum of squares (total - replicates - strains - replicates $\times$ strains interaction) divided by the degrees of freedom (total - replicates - strains replicates $\times$ strains interaction). In the example in Table $3(a)$ there would be 20 such matched pairs, of which 18 would have a variance of zero (being either both o or both I) and 2 with a variance of 0.5 . The mean variance would therefore be 0.05 , while by using the sums of squares from Table $3(c)$ one obtains the same answer in the form $(9 \cdot 9-8 \cdot 9) /(39-19)$. 


\section{Other statistical models}

The model discussed makes a number of simplifying assumptions that may not always be justified. It is assumed that the chance of mutation in replicates is inappreciable, as will usually be true. It is probably not true, however, that the probability, $p_{i 0}$, of a test being scored negative when it should be scored positive is equal to the probability, $p_{i 1}$, that it will be scored positive when it should be scored negative. Examples of this readily come to mind: many microbiologists would consider it more likely that Gram-positive organisms would be erroneously recorded as Gram-negative than the converse; the probability that an unpigmented strain is scored as producing pigment will generally be less than the probability that a pigmented strain will not show production of pigment. More generally, inequality of $p_{i 0}$ and $p_{i 1}$ will be shown by inequality of the proportion of scattered 'false negatives' (seen as entries where strains that are nearly always positive give occasional negative results) to the proportion of scattered 'false positives' (positive results obtained from predominantly negative strains). It would in theory be possible to calculate the variance separately for strains giving a majority of positive or of negative results and obtain the analogous averages over the tests, $p_{0}$ and $p_{1}$. From these one could derive

and

$$
\mathrm{E}\left(S^{\prime}\right) \simeq \frac{a}{n}\left(2 p_{1}^{2}-2 p_{1}+\mathrm{I}\right)+\frac{d}{n}\left(2 p_{0}^{2}-2 p_{0}+\mathrm{I}\right)+(\mathrm{I}-S)\left(p_{0}+p_{1}-2 p_{0} p_{1}\right)
$$

where

$$
\mathrm{SE}\left(S^{\prime}\right) \simeq \sqrt{(A+B)(\mathrm{I}-A-B) / n}
$$

and

$$
\begin{aligned}
& A=\left[2 a p_{1}\left(\mathrm{I}-p_{1}\right)+2 d p_{0}\left(\mathrm{I}-p_{0}\right)\right] / n \\
& B=(b+c)\left(p_{0}+p_{1}-2 p_{0} p_{1}\right) / n .
\end{aligned}
$$

In practice, however, it is uncertain whether there is a general tendency over tests as a whole for false negatives or false positives to be more frequent, and it seems likely that this more elaborate model will not bring gains commensurate with the greater calculation needed.

Similar considerations apply to the situation when the probability for strain $j, p_{j}$, is not equal to that for strain $k, p_{k}$. There are again reasons for expecting these to be different. One is a consequence of the inequality of $p_{0}$ and $p_{1}$; if one strain is from a taxon that scores positive in most tests and the other is from a taxon that scores negative in most tests this will make $p_{j}$ and $p_{k}$ unequal. The comparable equations are

and

$$
\mathrm{E}\left(S^{\prime}\right) \simeq S\left(2 p_{j}-\mathrm{I}\right)\left(2 p_{k}-\mathrm{I}\right)+p_{j}+p_{k}-2 p_{j} p_{k}
$$

where

$$
\begin{aligned}
\mathrm{SE}\left(S^{\prime}\right) & \simeq \sqrt{C(\mathrm{I}-C) / n} \\
C & =p_{j}\left(\mathrm{I}-p_{k}\right)+p_{k}\left(\mathrm{I}-p_{j}\right) .
\end{aligned}
$$

One would seldom have enough information to estimate $p_{j}$ and $p_{k}$ separately, and it is pointed out below that it appears to be difficult to demonstrate gross differences between taxa in this respect, though anecdotal evidence is sometimes heard that there are taxa with which it is very difficult to get reproducible results.

Expressions for $\mathrm{E}\left(S^{\prime}\right)$ and $\mathrm{SE}\left(S^{\prime}\right)$ for other coefficients, such as $S_{\mathrm{Y}}, S_{\mathrm{J}}$ and $S_{\mathrm{P}}$ (see Sokal \& Sneath, 1963; Sneath, 1968) are more complex and generally require also a knowledge of all the four quantities $a, b, c$ and $d$. When these coefficients are used it may be legitimate to take the values estimated for the coefficient $S_{\mathrm{SM}}$ as a rough guide in everyday practice. 


\section{RESULTS}

Earlier data from which to estimate test reproducibility are very limited. Results of Liston, Weibe \& Colwell (1963) suggest a value of $p$ of about $4 \%$ within one laboratory, since this would reduce the value of $S_{\mathrm{SM}}$ between subcultures of the same strain from unity to the observed average of about 0.92. Taylor et al. (1970) analysing data of Gottlieb (196I) reported that the reproducibility of tests commonly used for streptomycetes varied between laboratories from $63 \%$ to $97 \%$; their figures suggest, after correcting for degrees of freedom, $p$ of almost $20 \%$. Such data must be of limited value for taxonomy, because the expected similarity between identical strains would be only $69 \%$, while the standard error based on the 37 tests considered would be about $9 \%$. Lapage et al. (1970) obtained an average of $6.9 \%$ of discrepancies for replicate comparisons between laboratories for a conventional set of tests used to identify Gram-negative rods. This corresponds to $p$ of $3.9 \%$ if one assumes neither series is an accurate standard; in fact one series was better standardized than the other, so that $p$ may have been rather higher than this. Washington, $\mathrm{Yu} \&$ Martin (I97I) examined the agreement between microtests and conventional ones in Enterobacteriaceae, and one can calculate from their tables that there was a minimum of $4.2 \%$ of discrepancies after $24 \mathrm{~h}$ of incubation, and only a little less at $48 \mathrm{~h}$. In fields outside microbiology there is a little scattered evidence that $p$ may often be of the same order of magnitude. Thus in chemotaxonomic work on higher plants with the usual chromatographic techniques an examination of the data of Weimarck (1970) suggests that $p$ is around $5 \%$, while the analogue of discrepancies due to different times of reading is seen in the study of Taylor (197I), who found large changes in similarity values from leaf constituents over a period as short as one month.

The first set of results we report here consists of 82 tests done in duplicate by one of us (Johnson, 1972) on 2I strains received as belonging to the genera Achromobacter, Acinetobacter, Alcaligenes, Bordetella, Moraxella, Pasteurella and Yersinia. These tests were carried out on two separate occasions (in most cases about 6 months apart), in the same laboratory and employing the same medium, techniques and conventions for scoring. The inocula were comparable on the two occasions but were not specially standardized; they were from cultures freshly prepared from freeze-dried stocks. The tests, which were all scored positive (I) or negative (o), formed a representative set such as might be used in a numerical taxonomic study, and were in fact about two thirds of those used in such a study of Bordetella and related genera (Johnson, 1972), where full details of the test methods may be found. Table 4 shows the tests and their variances calculated by formula 15 . The number of tests with $0, \mathrm{I}, 2,3,4$ and 5 discrepancies were, respectively, 50, 20, 3, 5, 3 and I, giving a value of $s^{2}$ of 0.0168 , corresponding to $p$ of $\mathrm{I} \cdot 7 \mathrm{I} 3 \%$. However, I9 of the tests were invariant on both occasions in all the $2 \mathrm{I}$ strains (though almost all varied over the much longer set of strains in the main taxonomic study): it may perhaps be more meaningful to take the averages over the 63 tests that did vary with these 2 I strains, and for these tests $s^{2}$ is 0.0219 , equivalent to $p$ of $2 \cdot 242 \%$.

We are aware that this particular set of data may be somewhat biased by memory of how strains had reacted on the previous occasion; properly a blind trial should have been undertaken. Nevertheless, we believe that the figures given here have not greatly overestimated the test reproducibility, and represent reasonable averages for carefully performed tests within one laboratory.

We also investigated whether the reproducibility differed among different strains. There were few discrepancies in the 6 strains of Bordetella pertussis and B. parapertussis ( 3 strains 


\section{Table 4. Reproducibility in duplicate tests on strains of Bordetella and related genera}

The strains were those listed in Johnson ( I972) as JI, J3, J9, JI I, J23, J27, J3 I, J35, J42, J43, J55, J56, $\mathrm{J} 58, \mathrm{~J} 59, \mathrm{~J} 60, \mathrm{JI} 28, \mathrm{~J}$ 4 45, J 67 , J 78 , J I 8 I and $\mathrm{J} 2 \mathrm{II}$.

The tests are listed in ascending order of $s_{i}^{2}$, corresponding in turn to $0, \mathrm{I}, 2,3,4$ and 5 discrepancies. Nineteen of the tests, marked with an asterisk, were uniformly positive or negative on both occasions with all the $2 \mathrm{I}$ strains.

\section{Test}

Gram stain*, retention of methyl violet, shape or organisms, colony opaque, colony glossy,

colony regular, colony viscid, colony raised, diffusible pigment produced, colour of pigment, pigment from tyrosine, turbidity in broth, flaky sediment in broth*, viscous sediment in broth*, growth anaerobically, growth anaerobically with nitrate, requirements for $\mathrm{CO}_{2}{ }^{*}$, for $\mathrm{V}$ factor*, for $\mathrm{X}$ factor*, growth on nutrient agar, motility, growth with $0 \%$ $\mathrm{NaCl}$, growth with $3 \% \mathrm{NaCl}$, growth with $0.2 \%$ phenol, growth with $0.3 \%$ phenol*, growth with $0.4 \%$ phenol*, growth with $0.5 \%$ phenol $^{*}$, sensitivity to $\mathrm{O}_{\mathrm{I} 29}$, to thionin, to optochin*, to penicillin, to streptomycin*, to chloramphenicol*, to aureomycin*, to neomycin*, to nitrofurantoin, growth at $25^{\circ} \mathrm{C}^{*}$, at $30^{\circ} \mathrm{C}^{*}$, at $35^{\circ} \mathrm{C}^{*}$, catalase, hydrolysis of starch, of casein, egg-yolk reaction, production of $\mathrm{HCN}^{*}$, acid aerobically from $\mathrm{I} \%$ glucose, from $10 \%$ glucose, from $\mathrm{I} \%$ lactose*, from $10 \%$ lactose, acid anaerobically from $1 \%$ glucose, alkali aerobically from $\mathrm{I} \%$ lactose.

Pleomorphism, surface growth in broth, growth with $10 \%$ bile, growth with $4.5 \% \mathrm{NaCl}$, growth with $6 \% \mathrm{NaCl}$, growth with $0.1 \%$ phenol, sensitivity to basic fuchsin, to methyl violet, to safranine, to pyronin, to terramycin, to tetracycline, to sulphonamide, to novobiocin, growth at $15{ }^{\circ} \mathrm{C}$, oxidase, phosphatase, urease, hydrolysis of arginine, of tyrosine.

Growth with $5 \%$ bile, ammonia from peptone, nitrate reduction.

Haemolysis of horse blood, sensitivity to erythromycin, to oleandromycin, to 'Bactrim', $\mathrm{H}_{2} \mathrm{~S}$ production.

Organisms in irregular masses or sheets, organisms in chains or filaments, sensitivity to bacitracin.

Peroxidase.

Table 5. Strain differences in reproducibility of tests listed in Table 4: analysis of variance of discrepancies (excluding tests marked with an asterisk)

\begin{tabular}{lccccc}
\multicolumn{1}{c}{ Factor } & $\begin{array}{c}\text { Sums of } \\
\text { squares }\end{array}$ & $\begin{array}{c}\text { Degrees of } \\
\text { freedom }\end{array}$ & Variance & F ratio & Probability $(P)$ \\
Between tests & $4 \cdot 6002$ & 62 & 0.0742 & $\mathrm{I} \cdot 85$ & $<0.00 \mathrm{I}$ \\
Between strains & $\mathrm{I} \cdot 17 \mathrm{I} 16$ & 20 & 0.0586 & $\mathrm{I} \cdot 46$ & $0.05<P<0 . \mathrm{I}$ \\
Residual & $49 \cdot 6856$ & 1240 & 0.0401 & - & - \\
Total & 55.4574 & 1322 & - & - & -
\end{tabular}

of each species), averaging 0.5 per strain, while the average discrepancies per strain in the whole set of 2 I strains was $2 \cdot 76$. There was an excess of strains with low discrepancies, because the numbers of strains with $0,1,2,3,4,5$ and 6 discrepancies were respectively, 4, I, 5, 3, 4, 2 and 2. However, an analysis of variance (Table 5, but note this analysis is not of the same kind as Table 3) showed barely significant differences between strains, although the differences between tests are highly significant. In other words different tests show different reproducibilities, but it is not certain that different strains do. Similar difficulty in proving significant differences between reproducibility with different strains was found by the Report of the Pseudomonas Working Party (to be published), although there are good reasons for believing such differences must exist, as noted earlier.

The second set of data comes from unpublished records of the Pseudomonas Working Party kindly placed at our disposal by the Working Party, from which we have calculated some illustrative examples after first recoding the results into $\mathrm{I}$, o form. The findings are 
Table 6. Test reproducibility for pseudomonads between laboratories, within laboratories and at different times of reading

The values of $s_{i}^{2}$ were calculated from data collected by the Pseudomonas Working Party and reported on, together with the strains, media and methods, in its Report (to be published). For calculating $s_{i}^{2}$ the original readings were rescored as o and $\mathrm{I}$ in a manner consistent with usage for numerical taxonomy. Some data were rather scanty for statistical analysis but the results illustrate the approximate order of magnitude of the test errors under different circumstances.

\begin{tabular}{|c|c|c|c|}
\hline & \multicolumn{3}{|c|}{$s_{i}^{2}$ from matched readings } \\
\hline & $\begin{array}{l}\text { In different } \\
\text { laboratories }\end{array}$ & $\begin{array}{l}\text { In different } \\
\text { replicates } \\
\text { within } \\
\text { laboratories }\end{array}$ & $\begin{array}{l}\text { At different } \\
\text { times of } \\
\text { reading }\end{array}$ \\
\hline Motility (hanging drop) & $0.08 \mathrm{I} 2$ & 0.0079 & - \\
\hline Growth at $4{ }^{\circ} \mathrm{C}$ ( 7 and $\mathrm{I} 4$ days) & 0.0667 & - & 0.0458 \\
\hline Growth at $37^{\circ} \mathrm{C}$ ( 3 and 7 days) & 0.1750 & - & 0.0458 \\
\hline Growth at $42{ }^{\circ} \mathrm{C}$ (I and 3 days) & 0.1375 & - & 0.0125 \\
\hline Growth $\mathrm{pH}_{4} .5$ (3 and 7 days) & 0.1795 & - & 0.1239 \\
\hline Growth on Simmons's citrate (3 days) & 0.1587 & $0 \cdot 1270$ & - \\
\hline $\begin{array}{l}\text { Growth in denitrification medium } \\
\text { of Stanier et al. ( } 3 \text { and } 7 \text { days) }\end{array}$ & 0.1855 & 0.0119 & 0.0208 \\
\hline $\begin{array}{l}\text { Gas in denitrification medium of } \\
\text { Stanier et al. ( } 3 \text { and } 7 \text { days) }\end{array}$ & $0 \cdot 1548$ & 0.0536 & 0.0417 \\
\hline $\begin{array}{l}\text { Reduction of nitrate, peptone nitrate } \\
\text { medium ( } 3 \text { and } 7 \text { days) }\end{array}$ & 0.0808 & 0.0415 & 0.0446 \\
\hline $\begin{array}{l}\text { Gas from nitrate, peptone nitrate } \\
\text { medium ( } 3 \text { and } 7 \text { days) }\end{array}$ & 0.0499 & 0.0508 & 0.0204 \\
\hline Glucose, oxidation ( 1,3 and 7 days) & $0.049 \mathrm{I}$ & - & 0.0598 \\
\hline Glucose, fermentation ( 3 and 7 days) & 0.0 & - & 0.0 \\
\hline $\begin{array}{l}\text { Glucose, gas production ( } 3 \text { and } \\
7 \text { days) }\end{array}$ & 0.0128 & - & $0 \cdot 0$ \\
\hline Pyocyanin production ( 3 and 7 days) & 0.0210 & - & 0.0068 \\
\hline Fluorescin production ( 3 and 7 days) & 0.0734 & - & $0.02 \mathrm{I} 2$ \\
\hline $\begin{array}{l}\text { Acetic acid from ethanol, method of } \\
\text { Shimwell et al. ( } 3 \text { and } 7 \text { days) }\end{array}$ & 0.0900 & 0.0167 & 0.0500 \\
\hline $\begin{array}{l}\text { Acetic acid from ethanol, method } \\
\text { of Carr ( } 3 \text { and } 7 \text { days) }\end{array}$ & 0.0667 & 0.0167 & 0.2167 \\
\hline Aesculin hydrolysis ( 3 days) & 0.0326 & 0.0159 & - \\
\hline Arginine hydrolysis ( 3 and 7 days) & 0.0157 & 0.0060 & 0.0060 \\
\hline Urea hydrolysis $(6 \mathrm{~h})$ & $0 \cdot 1690$ & 0.0396 & - \\
\hline $\begin{array}{l}\text { Gelatin liquefaction, stab method } \\
\text { ( } 3 \text { and } 7 \text { days) }\end{array}$ & 0.0679 & 0.0166 & 0.0383 \\
\hline $\begin{array}{l}\text { Gelatin hydrolysis, plate method } \\
\text { ( } 3 \text { and } 7 \text { days) }\end{array}$ & $0 \cdot 1349$ & 0.0139 & 0.0238 \\
\hline Oxidase, Kovacs's method & 0.0701 & 0.0108 & - \\
\hline Gluconate ( 3 and 7 days) & $0.068 \mathrm{I}$ & 0.0244 & 0.0579 \\
\hline Egg yolk reaction (3 days) & 0.0988 & 0.0146 & - \\
\hline Melanin from tyrosine ( 3 and 7 days) & 0.0802 & 0.0060 & $0.042 \mathrm{I}$ \\
\hline
\end{tabular}

presented in Table 6, and illustrate two important points: (i) reproducibility between laboratories is usually a good deal worse than between replicates within laboratories; and (ii) some tests are very sensitive to differences in the time of reading. The reproducibility within laboratories for the I 7 tests on which data were available is given by the mean of $s_{i}^{2}$ as $s^{2}=0.0279$ (equivalent to $p$ of $2.87 \%$ ). This is close to the figure found for the first set of data.

In a few tests the reproducibility could be somewhat improved by adjusting the division between positive and negative. For example, the gluconate test was a little more consistent 
if a green reaction was considered positive (rather than scoring both blue and green as negative, and only a yellow precipitate as positive). Such adjustments, however, were not straightforward, because in many cases by carrying it to one or other extreme almost every strain became consistently positive or negative; the test then becomes valueless in practice, though its reproducibility is nominally good. Similarly, a test will appear highly reproducible if very few strains give positive or negative results. The reproducibility of a few tests in Tables 4 and 6 may have been overestimated for this reason. However, we do not think this is seriously misleading, because any set of tests used in practical work will exhibit a wide range of percent positive reactions, similar to those found in the two sets of data described above. It is true that by dividing $s_{i}^{2}$ by twice the proportion of positive or negative results, whichever is the less, one would obtain a figure that would in theory be the same as that from a set of strains half of which were positive and half negative. Yet a correction of this type rests on somewhat shaky foundations, for it is doubtful whether it is safe to assume that the set of strains actually used is comparable to a set deliberately chosen to have equal numbers of positive and negative strains. In addition, the resulting figure will have diminished statistical significance, for it may depend on the vagaries of results on very few strains.

If it were necessary to take the proportion of positive results into account it would be better to use an index such as $\left(\mathrm{I}-4 s_{i}^{2}\right) \times($ total sums of squares)/(total degrees of freedom), which would be a maximum when test error was zero and half the strains were consistently positive. It would become zero or negative if the test error was large or if the proportion of positive reactions was $O$ or $\mathrm{I}$. This index would be of most use in choosing the best tests for identification schemes (see Lapage \& Bascomb, I968); the first term would make allowance for test error, while the second term would pick out the most discriminatory tests in the same way as the separation index of Gyllenberg (1963).

\section{DISCUSSION}

The findings reported here show the practical importance of test error in numerical taxonomy. Test error causes loss of taxonomic structure, and, as can be seen from Fig. I, the resulting distortion becomes serious when the average probability of an incorrect test reading rises above $10 \%$. Nevertheless this source of taxonomic distortion must be judged against that due to sampling error, which predominates when the number of tests is small. Consequently, taxonomists should still use as many tests as possible provided the test error for any one is not too large. The upper limit to the permissible error on any one test is not yet clear, because a few unsatisfactory tests can evidently be tolerated. The ideal method would be to add the tests one by one, starting with the most reproducible, and find the point of lowest error as was done in Fig. 2, but for general guidance one might reject tests with more than 10 to $15 \%$ of error. Test error is most readily estimated by analysis of variance of replicates.

It is important to a worker to know the reproducibility of his tests within his own laboratory. Inattention to test error may well explain some unsatisfactory numerical taxonomic studies. If it is large enough such error could appear to destroy the difference even between extremely homogeneous and distinct species. Furthermore, this eventuality might not be recognized if there were no internal checks on the reliability of the techniques. For this reason it would seem sound practice to guard against the danger by replicating some of the strains in numerical taxonomic studies: one would then carry through replicate subcultures of selected strains as if they were separate strains. The replicates should in theory give identical results. In practice some differences will often appear (especially if the results on 
the replicates are read without reference to each other), and a rough but useful estimate of the test error can be obtained from the replicates by the methods described above (e.g. formula 15 ).

Other helpful information on reproducibility includes that on times of reading, such as the time that gives the most reproducible results. This need not, however, necessarily be that time which is best for taxonomic purposes (e.g. if all organisms are uniformly negative this is not very useful, as noted above). Although the effect of time of reading can be increased to almost any magnitude by taking very early and very late times, nevertheless many tests are conventionally read when the proportion of positive results is increasing rapidly. In such instances the effect of different times of reading may be worth knowing, because if the results are heavily dependent on this, one should take extra care to read at the specified time, e.g. at exactly $24 \mathrm{~h}$ and not 'at I day' which may mean $20 \mathrm{~h}$ or $26 \mathrm{~h}$. Similar considerations apply to accurate control of incubation temperatures. If the temperatures of the shelves of an incubator differ by even a small amount differences in growth rates may lead to some cultures being well in advance of others in their metabolic activities after several hours of growth. The effect of deliberately changing temperatures or times of reading is not the same as error in the usual sense, for it involves moving the strains systematically into another portion of taxonomic space, and not simply perturbing them erratically (an example on some strains of Listeria is given by Davis, Fomin, Wilson \& Newton I969). If the cause is unrecognized it may amount to the same thing in practice, although the use of pattern similarity coefficients (Sneath, 1968) helps to reduce problems of this kind.

The reproducibility of tests between laboratories must be expected to be generally larger than that within laboratories. This is because in addition to factors causing discrepancies within one laboratory (such as those just mentioned) there will be other sources of variation (for example, different batches or brands of culture media, differences of inoculation technique, and discrepancies between observers in reading the results). As with all variation of this kind, statistical errors will tend to accumulate rather than cancel one another. The reproducibility between laboratories is of some consequence in judging the likelihood of discrepancies in published work, but it would seem particularly important in collaborative studies. Despite certain drawbacks it might in such work be worth scoring the results into $\mathrm{I}$, o form in order to obtain rapid assessment of reliability by the methods proposed in this paper. One obvious conclusion, in view of the high variation commonly found between laboratories, is that it is best for each laboratory to carry out a limited number of tests, but to do them on all strains. Similar considerations could well apply to choosing tests that are best for identification (e.g. Lapage \& Bascomb, 1968) or to choosing the experimental conditions giving the clearest separation between taxa (e.g. Wayne, 1967) because in both situations test reliability is important. Much useful information can be obtained with little labour by even a modest number of replicates on a small set of strains.

In conclusion, two points may be made. Studies on experimental error must have implications for the certainty with which OTU's are positioned in taxonomic space, and hence for the position and extension of clusters that are thought to represent taxa (e.g. see Carmichael \& Sneath, 1969; Sneath, 1972); these implications deserve study, for they will be particularly important when the taxa are represented by few strains as is commonly the case. Also, the effects of test errors must be equally misleading for orthodox taxonomy, as well as for identification (whether conventional or numerical). They could be important also in other areas, such as clinical medicine, where little is known about the reliability or reproducibility of the primary data. 
We thank the members of the Pseudomonas Working Party of the Microbial Systematics Group of the Society for making available the detailed results of collaborative work. Much of the work was done during the tenure by one of us (R.J.) of a Medical Research Council Scholarship.

\section{REFERENCES}

Carmichael, J. W. \& Sneath, P. H. A. ( 1969). Taxometric maps. Systematic Zoology I8, 402-41 5.

Davis, G. H. G., Fomin, L., Wilson, E. \& Newton, K. G. (I969). Numerical taxonomy of Listeria, streptococci and possibly related bacteria. Journal of General Microbiology 57, 333-348.

FisHeR, D. R. \& RoHLF, F. J. (I969). Robustness of numerical taxonomic methods and errors in homology. Systematic Zoology 18, 33-36.

GoodalL, D. W. (1967). The distribution of the matching coefficient. Biometrics 23, 647-656.

GotTlieb, D. (I96I). An evaluation of criteria and procedures used in the description and characterization of the streptomycetes. A cooperative study. Applied Microbiology 9, 55-65.

GrewaL, M. S. (1962). The rate of genetic divergence of sublines in the C57BL strain of mice. Genetical Research 3, 226-237.

GylleNBERG, H. G. (1963). A general method for deriving determination schemes for random collections of microbial isolates. Annales Academiae Scientiarum Fennicae, series A IV (Biologica), No. 69, 23 pp.

Johnson, R. (1972). Aspects of the taxonomy of Bordetella and related organisms. Ph.D. Thesis, University of Leicester.

LAPAGE, S. P. \& BASCOMB, S. (1968). Use of selenite reduction in bacterial classification. Journal of Applied Bacteriology 3I, 568-580.

Lapage, S. P., Bascomb, S., Willcox, W. R. \& Curtis, M. A. (1970). Computer identification of bacteria. In Automation, Mechanization and Data Handling in Microbiology, pp. I-22. Edited by A. Baillie \& R. J. Gilbert. London: Academic Press.

Liston, J., Weibe, W. \& Colwell, R. R. (1963). Quantitative approach to the study of bacterial species. Journal of Bacteriology 85, 106I-1070.

LOCKHART, W. R. (1967). Factors affecting reproducibility of numerical classifications. Journal of Bacterio$\log y$ 94, 826-831.

Moroney, M. J. (1956). Facts from Figures, 3rd edn. Harmondsworth, Middlesex: Penguin Books.

OWen, D. B. (1962). Handbook of Statistical Tables. London: Pergamon Press.

SNEATH, P. H. A. (1968). Vigour and pattern in taxonomy. Journal of General Microbiology 54, I-I I.

SNEATH, P. H. A. (197I). Theoretical aspects of microbiological taxonomy. In Recent Advances in Microbiology, X International Congress for Microbiology, pp. 58I-586. Edited by A. Pérez-Miravete \& D. Peláez. Mexico, D.F.: Associacion Mexicana de Microbiologia.

SNEATH, P. H. A. (1972). Computer taxonomy. In Methods in Microbiology, vol. 7 A, pp. 29-98. Edited by J. R. Norris \& D. W. Ribbons, London: Academic Press.

SNedeCor, G. W. (1956). Statistical Methods applied to Experiments in Agriculture and Biology, 5th edn. Ames, Iowa: Iowa State University Press.

Sokal, R. R. \& Rohlf, F. J. (1969). Biometry, the Principles and Practice of Statistics in Biological Research. San Francisco: W. H. Freeman.

Sokal, R. R. \& Sneath, P. H. A. (1963). Principles of Numerical Taxonomy. San Francisco: W. H. Freeman.

TAyloR, G. R., Guthrie, R. K. \& ShiRling, E. B. (I970). Serological characteristics of Streptomyces species using cell wall immunizing antigens. Canadian Journal of Microbiology 16, 107-115.

TAYLOR, R. J. (197I). Intraindividual phenolic variation in the genus Tiarella (Saxifragaceae); its genetic regulation and application to systematics. Taxon 20, 467-472.

TAYLOR, R. J. \& CAMPBell, D. (1969). Biochemical systematics and phylogenetic interpretations in the genus Aquilegia. Evolution 23, 153-162.

Tremaine, J. H. \& Argyle, E. (1970). Cluster analysis of viral proteins. Phytopathology 6o, 654-659.

WASHington, J. A., YU, P. K. W. \& MARTIN, W. J. (I97I). Evaluation of accuracy of multitest micromethod system for identification of Enterobacteriaceae. Applied Microbiology 22, 267-269.

WAYNE, L. G. (1967). Selection of characters for an Adansonian analysis of mycobacterial taxonomy. Journal of Bacteriology 93, 1382-1391.

Weimarck, G. (1970). Spontaneous and induced variation in some chemical leaf constituents in Hierochloë (Gramineae), Botaniska Notiser 123, 231-268.

Williams, W. T. \& LANCE, G. N. (I969). Application of computer classification techniques to problems in land survey. Bulletin of the International Statistical Institute 42, 345-354. 